\title{
Sibirische Schwertlilie (Iris sibirica) - Blume des Jahres 2010
}

\author{
KuRT BAUMANN
}

\begin{abstract}
Siberian iris (Iris sibirica) is flower of the year 2010. It is endagered by the drainage of moist meadows. Distribution, morphology and flower biology are presented. Bumble bees are typical pollinators of Iris sibirica.
\end{abstract}

\section{Zusammenfassung}

Die Sibirische Schwertlilie (Iris sibirica) ist Blume des Jahres 2010. Aufgrund der Trockenlegung von Feuchtwiesen gehen ihre Bestände zurück und ist sie bedroht. Verbreitung, äußeres Erscheinungsbild und Blütenbiologie werden vorgestellt. Typische Bestäuber sind Hummeln.

\section{Name, Verbreitung und Standort}

„Blau, blau, blau blüht der Enzian“ - viele kennen wenigstens die erste Zeile dieses Liedes. Sie könnte aber bei Unkenntnis zu falschen Vorstellungen führen. Denn einerseits sind nicht alle Enzian-Arten blaublütig und anderseits gibt es eine ganze Reihe von Pflanzen, die blau blühen, aber keine Enziane sind. Von den blauen Blumen ist die Wegwarte, Blume des Jahres 2009, erwähnenswert. Auch die Blume des Jahres 2010, die Sibirische Schwertlilie (Iris sibirica), eine von 11 heimischen bzw. in Deutschland verwilderten Iris-Arten, hat besonders schöne blaue Blüten, weshalb sie deshalb manchmal auch Blaue Schwertlilie genannt wird. Diese Bezeichnung ist nicht eindeutig, existieren doch noch andere blau blühende Iris-Arten in Deutschland wie z. B. die Bastard-Schwertlilie (Iris spuria). Ihr Name ist völlig irreführend, da sie keine Kreuzung ist, weshalb neuerdings für sie der Name WiesenSchwertlilie vorgeschlagen wird. Um diesen Namen konkurriert $I$. spuria mit der Sibirischen Schwertlilie, die früher auch I. pratensis hieß. Der Gattungsname bezieht sich wegen der vielfältigen Farbenpracht mancher Arten auf die Göttin des Regenbogens (Iris).

Die Sibirische Schwertlilie ist außer der gelb blühenden Sumpf-Schwertlilie die in Deutschland am weitesten verbreitete Art. Es gibt archäologische Nachweise der Sibirischen Schwertlilie aus der späten Wärmezeit (vor 5000 2700 Jahren). LEONHARD FUCHS erwähnt sie in seinem Kräuterbuch von 1565. Die Vermutung, sie sei in Deutschland nach dem Mittelalter eingewandert oder als Gartenpflanze verwildert, gilt vielleicht für begrenzte Gebiete (BONSART 1960). Für den süddeutschen Raum wird angenommen, dass sie dort heimisch ist (GRIESE 1998). Sie wächst auch vereinzelt in Schleswig-Holstein, fehlt aber in Nordwest-Deutschland. Außerdem gedeiht sie im Bereich der Elbe, im Thüringer Raum, in Franken, an der Donau, im Ober- und Mittelrheintal, am Bodensee und im Voralpengebiet (HAEUPLER 1988). In unserer Region kann man sie beispielsweise in Rheinhessen und im Hessischen Ried antreffen. Als Zierpflanze ist sie in unseren Gärten und Parks weit verbreitet.

In Deutschland erreicht Iris sibirica die Westgrenze ihres Verbreitungsgebietes. Die Verbreitungsgrenze verläuft von der Lübecker Bucht über die obere Weser zur Moselmündung, über das Oberrheintal in das Elsass und zum Genfer See (EBERLE 1972). Isolierte Vorkommen sind für Westfrankreich und in Apulien nachgewiesen. Als eurasiatisches kontinentales Florenelement kommt sie von Mitteleuropa bis in das westsibirische Flachland bis zum Altai vor. Die früheren Angaben der Verbreitung bis nach Japan gelten für eine andere, von Iris sibirica abzugrenzende Art. Im Mittelmeerraum tritt sie nicht auf, ist aber von Norditalien nach SOEuropa (bis Bulgarien) verbreitet. Im Norden wächst sie meist südlich des 60 . Breitengrades, vereinzelt in Dänemark. Eingebürgert wurde sie im Osten der USA und in SO-Kanada.

Die Sibirische Schwertlilie gedeiht auf wechselfeuchten, mesotrophen Moorwiesen, d. h. auf periodisch überschwemmte Böden, die nicht zu nährstoffarm, mäßig kalk- und basenreich sind. Staunässe stört die Sibirische Schwertlilie ebenso 


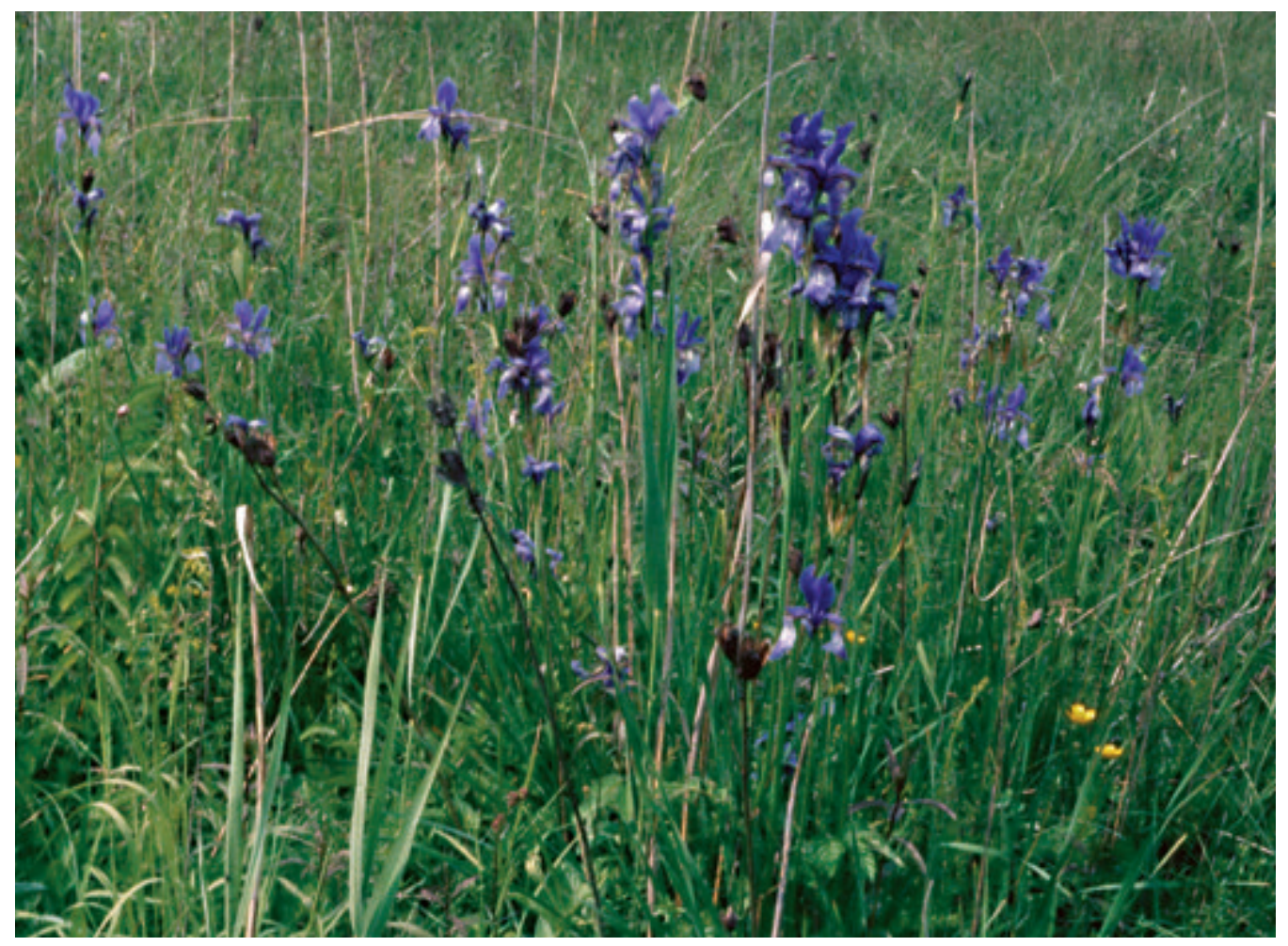

wenig wie Frost. Sie gedeiht bevorzugt auf Pfeifengraswiesen und moorige Waldwiesen sowie an Gräben. Wegen des Verlustes der Pfeifengraswiesen, die durch die Entwässerung stark zurückgedrängt werden, zählt die Sibirische Schwertlilie in Deutschland zu den stark gefährdeten Pflanzen und ist besonders geschützt.

\section{Habitus}

Die Sibirische Schwertlilie ist eine ausdauernde Staude. Sie entspringt einem nicht sehr dicken $(0,9-1,2 \mathrm{~cm})$, kurzen Wurzelstock, der sich nahe der Erdoberfläche befindet. Da sich dieser beim Wachsen verzweigt, kommt es zur Horstbildung (DüLL 2005). Aus dem Wurzelstock wächst im Frühjahr ein meist $60 \mathrm{~cm}$ hoher, hohler, runder und leicht abgeflachter Stängel. Am Grunde befinden sich als faserige Scheiden die Reste der Vorjahresblätter.

Die Laubblätter sind schmaler als bei den meisten anderen Iris-Arten und meist nur 2-6 mm breit. Sie sind zweizeilig angeordnet und kürzer als der Stängel. Ihre schmal lineale, abgeflachte
Form erinnert an ein Schwert. Die abgeflachten Blätter sind im oberen Teil scharfkantig und mit ihrem Grund umfassen sie den Stängel. Das Blatt ist nicht in eine Ober- bzw. -Unterseite differenziert. Ein Wachsüberzug macht die Blätter unbenetzbar. Das zur Photosynthese fähige Gewebe befindet sich auf beiden Seiten des Blattes.

\section{Blüten und Blütenbiologie}

Die Blütezeit liegt im Mai und Juni. Dann entwickelt sich ein traubiger Blütenstand mit 1-5 gestielten Blüten. Die Hoch- oder Tragblätter sind lanzettlich, braun und oberwärts trockenhäutig. Die äußeren drei Perigonblätter sind elliptisch bis verkehrt eiförmig und herabgebogen. Diese auch als Hängeblätter bezeichneten Blütenblätter dienen zur Anlockung und als Landeplatz für die Bestäuber. Ihre breite, $3-6 \mathrm{~cm}$ lange Platte (vorderer Teil des Blütenblattes) ist hell violett bis weiß gefärbt und zeigt dunkel-

Abb. 1: Wiese mit Iris sibirica. 
blau-violette Adern sowie ein gelbes Saftmal. Bärtige Strukturen wie bei anderen Iris-Arten fehlen. Die farbigen Flächen, die wie ein Staubblatt wirken (BARTH 1982) sowie ein angenehmer Duft locken die Bestäuber effektiv an. Die breite Platte der Hängeblätter verschmälert sich abrupt in den unteren Teil des Blütenblattes. Dieser Bereich ist bräunlichgelb gefärbt mit rotpurpurner Aderung. Im unteren Teil sind die Perigonblätter zu einer langen Röhre verwachsen, in der Nektar abgeschieden wird. Die inneren drei Perigonblätter sind dunkler violett gefärbt als die äußeren und stehen aufrecht. Da sie bei manchen Iris-Arten so gebogen sind, dass sie sich berühren, werden sie Domblätter genannt.

Schwertlilien haben einen dreifächerigen, unterständigen Fruchtknoten und nur drei Staubblätter. Diese liegen den blütenblattähnlichen, dunkelvioletten Griffelästen an, die den äußeren Perigonblättern dachartig gegenüber stehen. Als Narbe dient nur ein winzig kleines, unauffälliges Läppchen am Ende der Unterseite des Griffelastes oberhalb des Staubblattes. Nur die normalerweise an den Griffelast geschmiegte Oberseite des Narbenläppchens ist belegungsfähig. Selbstbestäubung ist hier räumlich ausgeschlossen.

Über die Blütenbiologie der Sibirischen Schwertlilie ist nichts Genaueres bekannt. Da der Blütenbau desjenigen der Sumpf-Schwertlilie (Iris pseudacorus) entspricht, wird angenommen, dass auch die Bestäubungsvorgänge gleich sind (Hegr 1939). Der Nektar wird nur an der Basis der äußeren Perigonblätter in der Blütenröhre abgeschieden, weshalb nur langrüsselige Insekten (Hummeln und manche Schwebfliegen) als Bestäuber in Frage kommen (HESs 1983). Da diese beiden Insektengruppen sehr verschieden sind, wurden früher zwei Blütenvarietäten postuliert: die eine für die dicken Hummeln mit einem breiten Eingang zwischen äußerem Perigonblatt und dem Griffelast, die andere für die Schwebfliegen mit einer schmalen Öffnung. Inzwischen

Abb. 2 (oben): Blüte von Iris sibirica.

Abb. 3 (unten): Blüte von Iris germanica, stellvertretend für den allgemeinen Bau einer Iris-Blüte; hier eine Teilblüte.
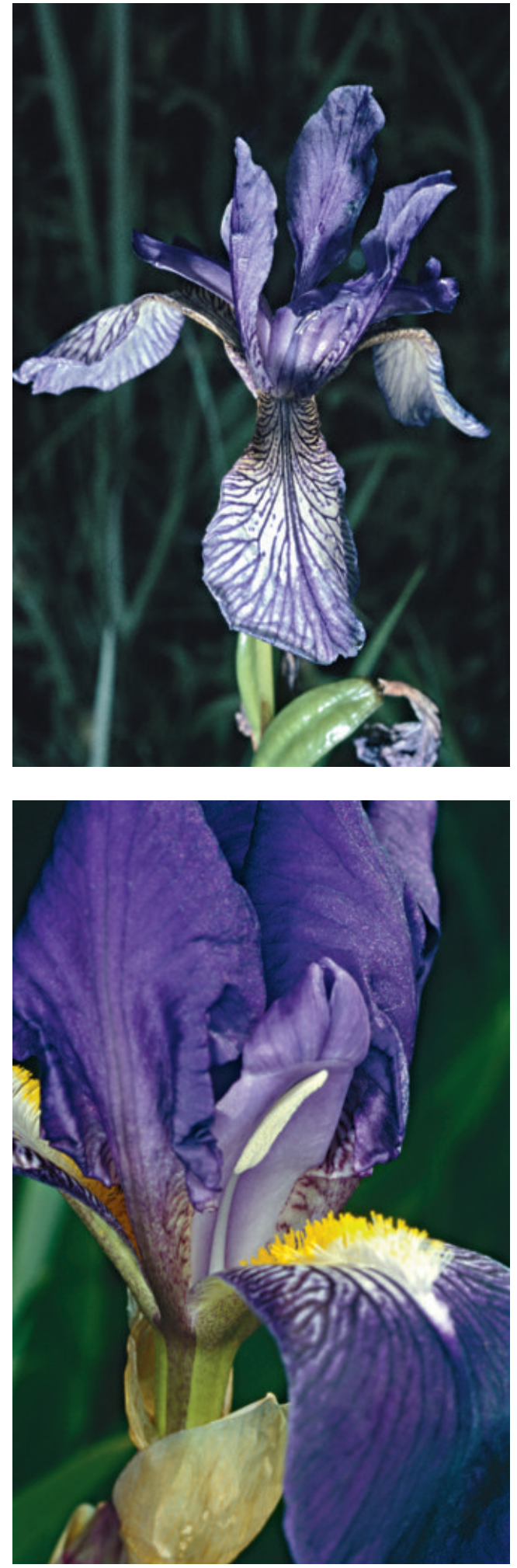
wurde erkannt, dass diese Unterschiede auf Altersunterschieden einer Blüte beruhen. Im jungen Zustand existiert ein breiter Blüten-Zugang, der sich später durch das Herabsenken des Griffelastes verschmälert.

Die Hummel kriecht, dem Saftmal folgend, in den Eingang zwischen Perigonblatt und Griffelast. Dabei klappt sie den Narbenlappen nach unten, so dass dessen belegungsfähige Oberseite zum Insekt gewendet ist. Bringt die Hummel Pollen einer anderen Iris-Blüte mit, kann er auf der Narbe abgelegt werden. Hat die Hummel in der Röhre den Nektar aufgesaugt und bewegt sich nun rückwärts nach oben, kann sie den Pollen aufnehmen. Sie klappt beim Hochkriechen den Narbenlappen nach oben, so dass nur die sterile Unterseite mit dem eigenen Pollen in Berührung kommt und eine Selbstbestäubung ausbleibt. Der Griffelast mit der Narbe, das Staubblatt und das äußere Perigonblatt sind bestäubungsökologisch funktionell eine Einheit, die botanisch als Blume bezeichnet wird. Nach dieser Definition besteht eine Iris-Blüte aus drei

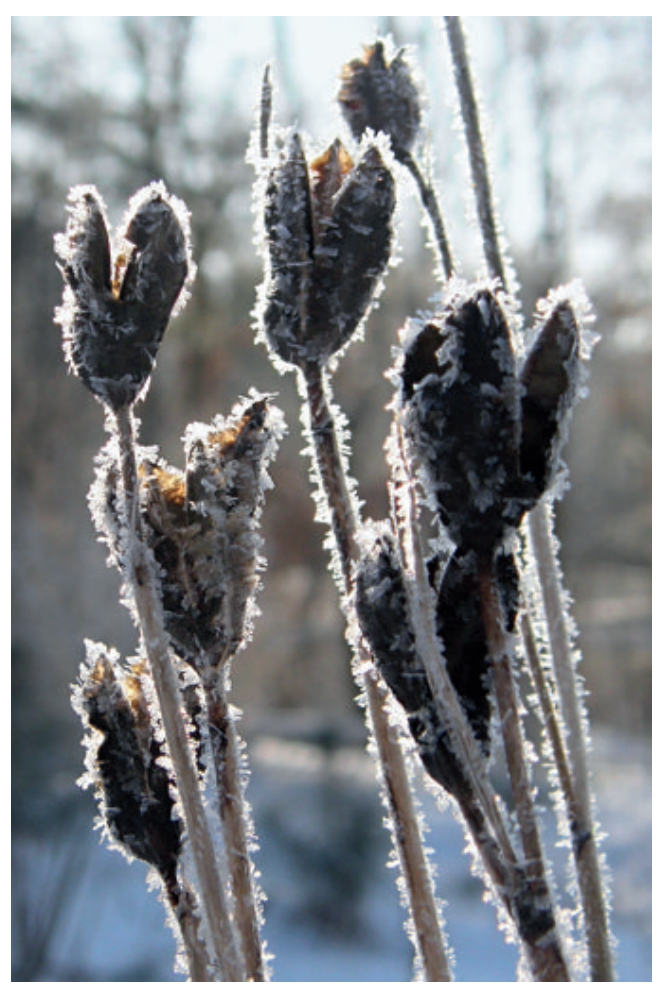

Einzelblumen, die nach dem Bau als Rachenblumen angesehen werden.

Fliegt eine bestäubende Hummel zur nächsten Teilblume einer Iris-Blüte, könnte es theoretisch zu einer Selbstbestäubung kommen. Dies wird aber ausgeschlossen, da Iris selbststeril ist (HESs 1983). Zudem sind Iris-Blüten vormännlich, so dass zunächst die Staubbeutel geöffnet sind, während die Narbenlappen noch nicht umgeklappt werden können und somit noch nicht empfängnisbereit sind.

Nach der Befruchtung wächst eine 3-4 cm lange dreifächerige Kapsel heran. Sie öffnet sich im September oder Oktober an den Mittelrippen (LEINS \& ERBAR 2008). Die elastischen Stängel des Fruchtstandes bewegen sich bei Berührung oder im Wind, so dass die zahlreichen abgeflachten Samen ausgestreut werden. Die Samen keimen leicht und nach drei Jahren blühen die Pflanzen erstmals.

\section{Literatur}

BARTH, F.G. 1982: Biologie einer Begegnung. - Stuttgart. BONSART, v. H.1960: Geschichtliches und Geschichten von Blumen. - Kosmos 56: 242-244.

Düll, R \& KuTZELnigg, H. 2005: Taschenlexikon der Pflanzen Deutschlands. 6. Aufl. - Wiebelsheim.

EberLe, G. 1972: Lilien und Lilienverwandte. - Frankfurt. GRIESE, J. 1998: Iridauae, Schwertliliengewächse. - In: Sebald, O. Seybold, S. \& Philippi, G.: Die Farm- und Blütenpflanzen Baden-Württembergs. - Stuttgart. Haeupler, H. \& SCHÖNfelder, P. 1988: Atlas der Farn- und Blütenpflanzen der Bundesrepublik Deutschland. - Stuttgart.

HEGI, G. 1939: Illustrierte Flora von Mittel-Europa. Bd. II. 2. Aufl. - München.

Hess, D. 1983: Die Blüte. - Stuttgart.

LeINS, P. \& ERbar, C. 2008: Blüte und Frucht. 2. Aufl. - Stuttgart.

Abb. 4: Vereiste Fruchtstände von Iris sibirica im Ökologisch-Botanischen Garten von Bayreuth. 